Prepared for the U.S. Department of Energy

under Contract DE-AC05-76RL01830

Work performed for the

Office of Defense Nuclear Nonproliferation (NA-20)

Office of Nonproliferation Research and Development (NA-22)

\title{
Electromagnetic material changes for remote detection and monitoring: a feasibility study
}

\section{Progress report}

\author{
JS McCloy \\ D Jordan \\ JF Kelly \\ DL McMakin \\ BR Johnson \\ LW Campbell
}

September 2009

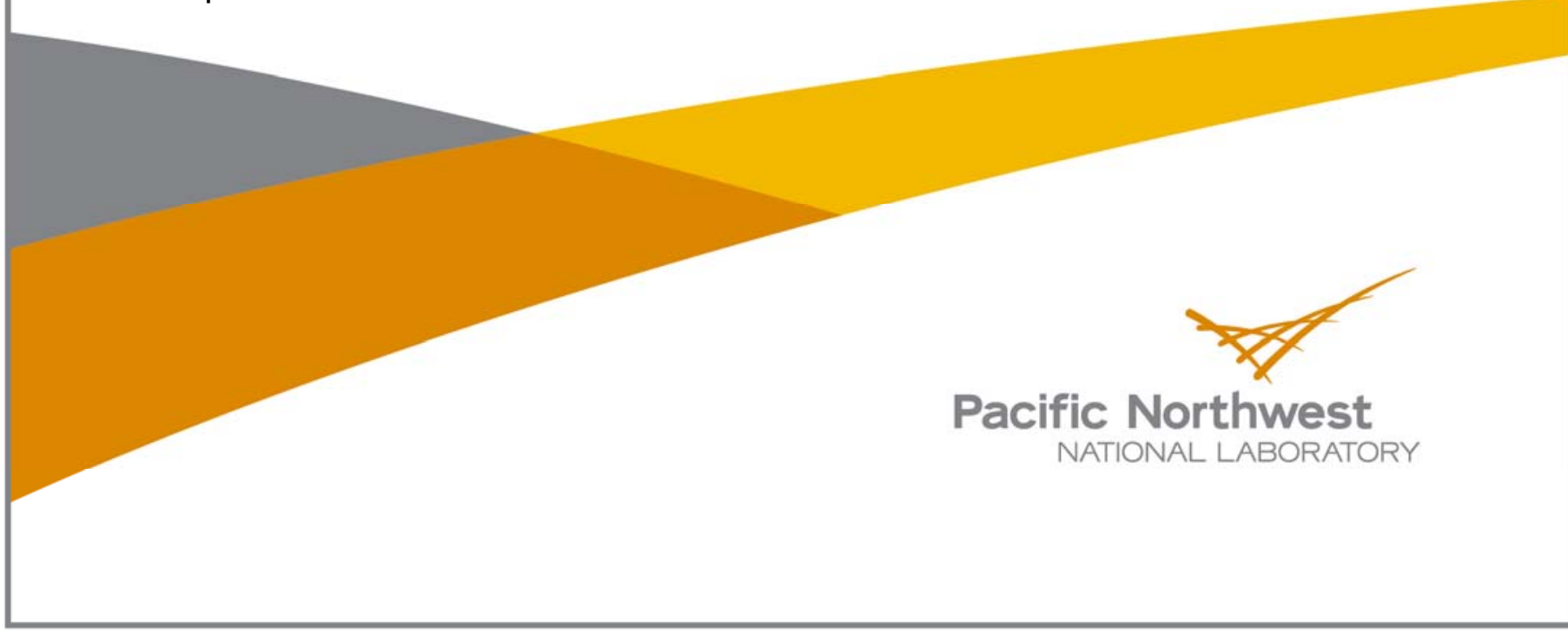




\title{
DISCLAIMER
}

This report was prepared as an account of work sponsored by an agency of the United States Government. Neither the United States Government nor any agency thereof, nor Battelle Memorial Institute, nor any of their employees, makes any warranty, express or implied, or assumes any legal liability or responsibility for the accuracy, completeness, or usefulness of any information, apparatus, product, or process disclosed, or represents that its use would not infringe privately owned rights. Reference herein to any specific commercial product, process, or service by trade name, trademark, manufacturer, or otherwise does not necessarily constitute or imply its endorsement, recommendation, or favoring by the United States Government or any agency thereof, or Battelle Memorial Institute. The views and opinions of authors expressed herein do not necessarily state or reflect those of the United States Government or any agency thereof.

\author{
PACIFIC NORTHWEST NATIONAL LABORATORY \\ operated by \\ BATTELLE \\ for the \\ UNITED STATES DEPARTMENT OF ENERGY \\ under Contract DE-AC05-76RL01830
}

Printed in the United States of America

Available to DOE and DOE contractors from the

Office of Scientific and Technical Information,

P.O. Box 62, Oak Ridge, TN 37831-0062;

ph: (865) 576-8401

fax: $(865) 576-5728$

email: reports@adonis.osti.gov

\author{
Available to the public from the National Technical Information Service, \\ U.S. Department of Commerce, 5285 Port Royal Rd., Springfield, VA 22161 \\ ph: (800) 553-6847 \\ fax: (703) 605-6900 \\ email: orders@ntis.fedworld.gov \\ online ordering: http://www.ntis.gov/ordering.htm
}

This document was printed on recycled paper.

$(9 / 2003)$ 


\title{
Electromagnetic material changes for remote detection and monitoring: a feasibility study
}

\section{Progress report}

\author{
JS McCloy \\ D Jordan \\ JF Kelly \\ DL McMakin \\ BR Johnson \\ LW Campbell
}

Prepared for

the U.S. Department of Energy

under Contract DE-AC05-76RL01830

Pacific Northwest National Laboratory

Richland, Washington 99354 


\section{Executive summary}

A new concept for radiation detection is proposed, allowing a decoupling of the sensing medium and the readout. An electromagnetic material, such as a magnetic ceramic ferrite, is placed near a source to be tracked such as a shipping container. The electromagnetic material changes its properties, in this case its magnetic permeability, as a function of radiation. This change is evident as a change in reflection frequency and magnitude when probed using a microwave/millimeter-wave source. This brief report discusses modeling of radiation interaction of various candidate materials using a radiation detector modeling code Geant4, system design considerations for the remote readout, and some theory of the material interaction physics. The theory of radiation change in doped magnetic insulator ferrites such as yttrium iron garnet (YIG) seems well founded based on literature documentation of the photomagnetic effect. The literature also suggests sensitivity of permittivity to neutrons in some ferroelectrics. Research to date indicates that experimental demonstration of these effects in the context of radiation detection is warranted.

\section{Table of Contents}

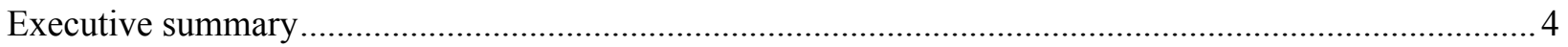

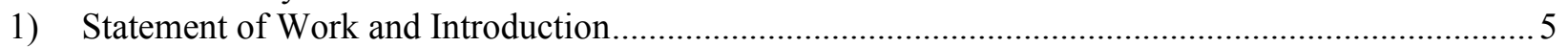

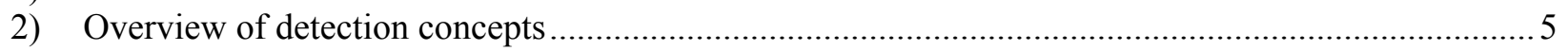

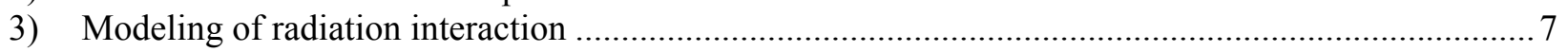

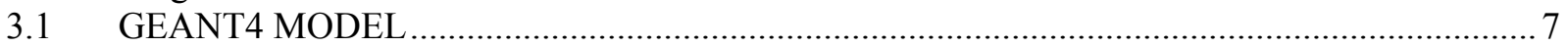

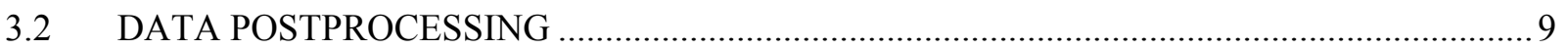

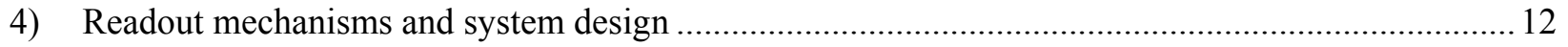

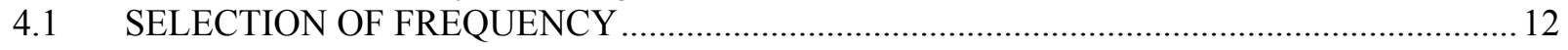

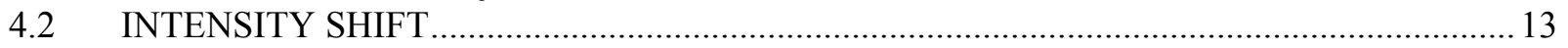

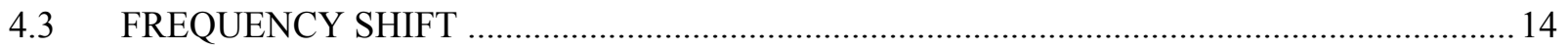

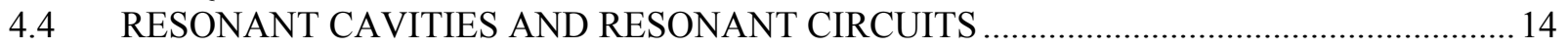

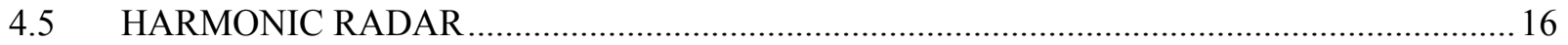

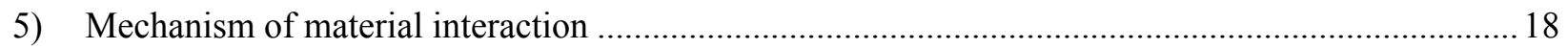

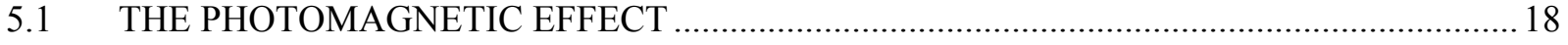

6) Interim conclusions, unanswered questions, and future plans ...................................................... 19

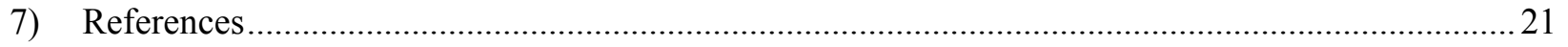




\section{1) Statement of Work and Introduction}

PNNL will perform an initial evaluation and limited proof-of-principle testing to assess the feasibility of using electromagnetic materials in stand-off radiation detection. The intention for this initial evaluation is to perform analysis and modeling of 1) resonant cavity considerations, 2) split ring resonator design, 3) nuclear interactions, and 4) expected response. Additionally, a detailed consideration of key experiments and required equipment and configurations will be generated. Some key items and materials may be procured for this round of initial testing.

Deliverable: - PNNL sends a report addressing feasibility to NA-22. The intent is to provide NA-22 with the requisite technical information to make an informed decision regarding continued detailed development of this technology.

This report is seen as the first of two reports summarizing the activities of this project. The intent of this current report is to generate dialogue with NA-22 on additional evidence desired in order to properly evaluate the potential of this technology.

This report is organized as follows.

- A summary of radiation detection concept-of-operations (CONOPS)

- A summary of the radiation interaction with the detector material explored using Geant4 modeling on some electromagnetic materials of interest

- A summary of the system considerations of the readout scheme including: 1) operational frequency selection, modes of operation including 2) intensity shift mode and 3) frequency shift mode, 4) discussion of resonant circuits and split ring resonators, and 5) discussion of harmonic radar

- A brief introduction to one possible physical mechanism for an observable electromagnetic property change, the photomagnetic effect

- Some interim conclusions and future plans

\section{2) Overview of detection concepts}

It was desired to explore the potential for use of a diverse set of electromagnetic materials in a new radiation detector context. That is, a secondary effect of radiation induced in a nearby "active" material would change the electromagnetic properties of the material (dielectric permittivity, electrical conductivity and/or magnetic permeability) to a sufficient extent that its bulk electromagnetic response would be viewable from substantial standoff distances using standard radar techniques.

The advantage of this concept over traditional radiation detection is that the detector and readout are decoupled. Hence, the detector can be very close to the radiation source (such as part of a "tag" for shipping containers, storage containers, or covertly placed), and the readout mechanism can be at a substantial standoff (i.e. at least $1 \mathrm{~km}$ ). Using a secondary signal of radiation, viewable in the 1 to $\sim 200$ $\mathrm{GHz}$ range where the atmosphere is very transparent (see Section 4.1), the range of detection becomes a matter of transmitter, receiver, and antenna parameters (see Section $4.3-4.5$ ), as well as the strength of the material interaction (see Section 4.2,5). This is a way to get around the classic problem of radiation detection. That is, $99.9 \%$ of $1 \mathrm{MeV}$ gamma photons get through 1 meter of air without scattering while only $5 \times 10^{-4}(0.05 \%)$ of $1 \mathrm{MeV}$ gamma photons get through 1 kilometer of air before being scattered to a different direction and/or losing energy to Compton scattering, the photoelectric effect, or pair production. 
The readout consists of viewing one or more of the following (see Figure 1):

1) Intensity shift (reflection change) due to change in conductivity, permittivity, or permeability

2) Frequency shift of reflection due to a change in frequency of a fundamental material resonance. The frequency shift can further be divided into one of several modes:

a) shift in the frequency of the reflected energy from the free space propagated wave incident on bare active material due to radiation induced changes of that materials electromagnetic properties

b) frequency change and/or Q factor change of a resonant cavity containing the active material

c) frequency change of a harmonic radar return signal due to an active material in the circuit of the tag antenna which changes its inductance, capacitance, or resistance when exposed to radiation.

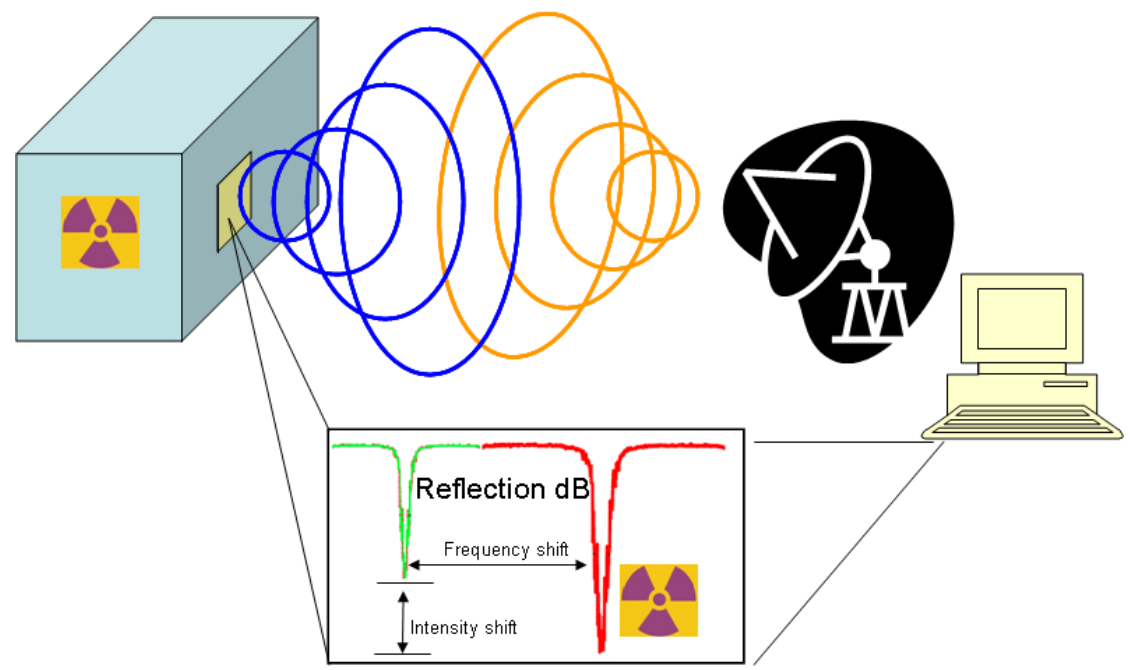

Figure 1: Concept for stand-off detection of radiation using secondary effects in electromagnetic materials

Note that 1) has previously been demonstrated by Tepper and Lossee [1] in a waveguided resonant cavity containing cryogenically cooled high purity Ge or CZT, where they detected single $4 \mathrm{MeV}$ x-ray photons by monitoring reflected power at 4-10 GHz. Through the change a resonant cavity frequency and Q factor they were able to deduce the radiation-induced change in conductivity. We postulate that the variations of observing a frequency change (mode 2) will be more sensitive and more likely to be useful at room temperature and at standoff distances.

Depending on the activity of the radiation source and the lifetimes of the carrier-mediated electromagnetic response, the detector can function in pulse counting or constant current mode (see Section 3.2). The magnitude and the kinetics of the electromagnetic response to changes in free-carrier population will be the focus of experimental investigation.

The envisioned physics in the detector material(s) is that the gamma photons will interact with the material creating electron-hole pairs. A primary effect will be the decrease in resistivity due to the creation of free electrons. If the material is a semiconductor this will have a non-negligible effect on the overall material response whereas in an insulator it will likely be a secondary effect (such as on the dielectric loss). In magnetic materials, additional effects are expected based on the photomagnetic effect (e.g. [2-4], see Section 4). Similar effects of radiation on frequency change are anticipated for ferroelectrics which have domain or grain resonances in the $1-10 \mathrm{GHz}$ range $[5,6]$. There has been indication in the literature that the permittivity of some ferroelectrics is sensitive to neutron exposure (e.g. $[7,8]$ ). Ferroelectric (ferromagnetic) materials are active to radiation since the polarization (magnetization) is a product of the number of electric (magnetic) dipoles and the electric (magnetic) dipole moment. One or the other or both of these quantities is anticipated to change as the result of 
ionizing radiation and creation of free electrons. Polarization (magnetization) is known to be related to dielectric permittivity (magnetic permeability).

The current assumption is that the "active" electromagnetic material would be accessible to interrogating radar, that is, not inside a metal container. This could be accomplished by having a "tag" similar to an RF tag on the outside of a metal box or on the inside of a polymer composite box. Metals have a very small skin depth and so electromagnetic waves of low energy do not pass through them but are attenuated. Additional potential applications could involve covert application of "sensor paints" containing dispersed particles onto surfaces which could then be probed remotely [9].

\section{3) Modeling of radiation interaction}

In this section the radiation interaction simulations of several candidate electromagnetic materials, both ferrimagnetic and ferroelectric, are discussed. Essentially the output of the model was probability of interaction and electron-hole pairs produced.

\subsection{GEANT4 MODEL}

Because the assumption in this scenario is that the material is very proximal to the radiation source, such as inside a shipping container to which the "active" material is affixed, a small distance from the source to the material $(5 \mathrm{~cm})$ was assumed in the Geant 4 modeling below. This should not be confused with the anticipated range of the overall radiation detection technique, which relies on the response generated in the material. The active volume was chosen as $0.1 \mathrm{~cm}^{3}(1 \times 1 \times 0.1 \mathrm{~cm}$ thick $)$ as a representative sample size that could be measured and compared with results.

PNNL simulated the response of several candidate materials to mono-energetic gamma rays using the Geant4 radiation transport detector modeling framework [10]. The main purpose of the simulation was to compute the deposited-energy spectrum in the material sample. The Geant 4 package supports modeling of the cascade of secondary electrons and photons generated by the primary gamma-ray's interaction, and thus provides a means of computing ionization energy deposition. The Geant 4 collaboration claims that its "low-energy" electromagnetic interaction processes are valid down to $250 \mathrm{eV}$, i.e. two orders of magnitude larger than the band gap of typical materials ( $\sim \mathrm{few} \mathrm{eV})$. Thus Geant4 does not directly support detailed modeling of features and interactions (e.g. band structure, hole transport, collective excitations, etc.) of greatest relevance to condensed-matter and materials science applications. However, in combination with literature values of the band gap, the Geant 4 energy-deposition spectrum can be used to obtain a rapid and reasonably accurate estimate of the spectrum of the number of electron-hole (e- h) pairs produced in the material. The simulation's (electron, photon)-cascade tracking capability can also provide information on the spatial distribution and density of $(\mathrm{e}, \mathrm{h})$ pairs produced in the material, although the main computational emphasis to date has been a simple estimate of the number of pairs produced. ${ }^{1}$

The Geant 4 simulations were run as follows: For each combination of (material, gamma-ray energy), $10^{7}$ gamma-rays were emitted isotropically from a point source located $5 \mathrm{~cm}$ away from the center of the $1 \times 1 \times 0.1 \mathrm{~cm}^{3}$ sample. The emission point was on the axis perpendicular to the $1 \times 1 \mathrm{~cm}^{2}$ sample face. Geant4 "low energy" electromagnetic-interaction physics models were enabled for the gamma-ray tracking; physics processes include photoelectric effect, Compton scattering, and pair production for gamma-rays, ionization and bremsstrahlung for secondary electrons and positrons, and annihilation for

\footnotetext{
${ }^{1}$ A Monte Carlo code recently developed at PNNL, NWEGRIM, can be applied to the modeling of the impact ionization cascade down to the production of $(e, h)$ pairs, but requires detailed information on interaction cross sections specific to the material of interest and is computationally expensive to run at incident energies above $\sim 100 \mathrm{keV}$. See [11]
} 
positrons. Table 1 displays the material properties assumed for the simulations. The materials were chosen as a set of representative ones under consideration. Incident gamma-ray energies were selected to span the range of greatest relevance to passive detection in threat-reduction applications, and to roughly represent emissions from common and/or significant background, industrial, and special nuclear material (SNM) sources. Table 2 displays the list of energies modeled and the corresponding motivation.

Table I: Material parameters assumed for the Geant 4 gamma-ray response simulations

\begin{tabular}{|c|c|c|c|c|}
\hline Material & $\begin{array}{l}\text { Compound } \\
\text { Formula }\end{array}$ & $\begin{array}{l}\text { Density } \\
\left(\mathrm{g} / \mathrm{cm}^{3}\right)\end{array}$ & $\begin{array}{l}\text { Band Gap } \\
\text { used }(\mathrm{eV})\end{array}$ & $\begin{array}{l}\text { Literature } \\
\text { range }(\mathrm{eV})\end{array}$ \\
\hline YIG (Yttrium Iron Garnet) & $\mathrm{Y}_{3} \mathrm{Fe}_{5} \mathrm{O}_{12}$ & 5.17 & 2.7 & $2.6-2.9$ \\
\hline GIG (Gadolinium Iron Garnet) & $\mathrm{Gd}_{3} \mathrm{Fe}_{5} \mathrm{O}_{12}$ & 6.44 & 2.5 & $2.5-3.0$ \\
\hline $\begin{array}{ll}\text { CMT (Cadmium } & \text { Manganese } \\
\text { Telluride) } & \\
\end{array}$ & $\mathrm{Cd}_{0.55} \mathrm{Mn}_{0.45} \mathrm{Te}$ & 6 & 2.1 & \\
\hline LN (Lithium niobate) & LiNbO3 & 4.64 & 4.0 & $3.6-4.8$ \\
\hline LT (Lithium tantalate) & LiTaO3 & 7.46 & 3.9 & $3.9-4.5$ \\
\hline NF (Nickel ferrite or trevorite) & $\mathrm{NiFe}_{2} \mathrm{O}_{4}$ & 5.38 & 2.2 & $1.1-5.0$ \\
\hline
\end{tabular}

Simulation output consists of a histogram of energy deposition in the sample. The distribution in the number of (electron, hole) pairs per gamma-ray incident on the material sample surface is derived from the energy-deposition histograms by the following procedure:

- Convert the histogram bin labeling from energy deposited to number of $(e, h)$ pairs by dividing the energy deposition by the band gap;

- Divide the number of events recorded in each bin by the number of gammas thrown and a geometrical factor $\left(=\mathrm{A}_{\text {sample }} / 4 \pi \mathrm{D}_{\text {model }}{ }^{2}\right.$, where $\mathrm{A}_{\text {sample }}\left(=1 \mathrm{~cm}^{2}\right)$ is the sample face area and $\mathrm{D}_{\text {model }}$ $(=5 \mathrm{~cm})$ is the modeled source-to-sample standoff distance) to take into account the solid angle subtended by the material sample relative to the point gamma-ray source.

The resulting distribution is the probability for generation of a specified number of (e,h) pairs, $\mathrm{N}_{\mathrm{e}, \mathrm{h}}$, falling within a bin of width $\Delta \mathrm{E}_{\text {dep }} / \mathrm{E}_{\text {gap }}$ (where $\mathrm{E}_{\text {dep }}$ is the energy deposited in the material and $\mathrm{E}_{\text {gap }}$ is the band gap), when a gamma-ray of energy $\mathrm{E}_{\gamma}$ is incident on the surface of the material, averaged over angles of gamma-ray incidence for a source-point relatively close to the material. The $\mathrm{N}_{\mathrm{e}, \mathrm{h}}$ spectrum at larger standoff distances, $\mathrm{D}$, can be estimated from the modeled results at $5-\mathrm{cm}$ standoff by multiplying by the factor $\left(A_{\text {sample }} / 4 \pi D^{2}\right) f_{\text {atten }}\left(E_{\gamma}\right)$, where $f_{\text {atten }}$ is an energy-dependent factor representing attenuation of the incident gamma-ray in all material media (e.g. air) interposed between the source point and the material sample.

Table II: Gamma-ray energies modeled in Geant4 simulations

\begin{tabular}{|l|l|l|}
\hline $\mathrm{E}_{\gamma}(\mathrm{keV})$ & Isotope & Relevance \\
\hline 60 & Am-241 & $\mathrm{SNM}$ \\
\hline 100 & low-energy range for U & $\mathrm{SNM}$ \\
\hline 300 & $\begin{array}{l}\text { mid-range between 186 keV (U-235) } \\
\text { and }(376,414) \mathrm{keV}(\mathrm{Pu}-239)\end{array}$ & $\mathrm{SNM}$ \\
\hline 662 & Cs-137 & $\begin{array}{l}\text { Industrial source; ubiquitous detector } \\
\text { benchmark }\end{array}$ \\
\hline 1460 & $\mathrm{~K}-40$ & Major background component \\
\hline 2614 & Th-232 decay chain & Background \\
\hline
\end{tabular}




\subsection{DATA POSTPROCESSING}

Figure 2a illustrates a "pulse-amplitude spectrum" in terms of binned electron hole pairs as generated in one of the materials (YIG) as a function of incident photon energy. The photopeak and Compton edge can clearly be seen at incident gamma-ray energies below about $662 \mathrm{keV}$. Figure $2 \mathrm{~b}$ is the same data viewed as a bar chart. In this case the x-axis is a category (bin) axis and so does not contain evenly spaced numbers of electrons, but more clearly shows the photopeaks associated with the characteristic photons. At higher energies $(\sim 1 \mathrm{MeV}$ and above), the probability of full energy deposition in these relatively thin material samples is negligibly small. Thus the distribution in (electron, hole) pair production is likely to reflect characteristic features of the incident spectrum for energies in the fewhundred keV range, of particular relevance to SNM detection (excluding the 1001-keV gamma-ray from U-238). Higher-energy sources, including common background components such as K-40 and Th-232, are likely to contribute a relatively featureless ("flat") background. These simulations suggest the appropriate scale in carrier production for one concept-of-operations of the proposed technique. The signal processing method (e.g. integration times) and carrier dynamics in the material (i.e. lifetimes as they relate to the secondary effect observed, such as magnetic moment change) must facilitate sensitive signal response over this carrier range in order for a meaningful pulse-amplitude spectrum to be feasible. The ultimate energy resolution achievable in the few-hundred $\mathrm{keV}$ regime will of course depend on the detailed relationship between the number of (and possibly the spatial distribution of) electron-hole pairs produced and the intensity of the signal produced. The possibility of registering cleanly-separated (in time) responses from single gamma-ray events, an essential requirement for accumulating a meaningful pulse-height spectrum, also depends on the total intensity of radiation incident on the material, as discussed further below.
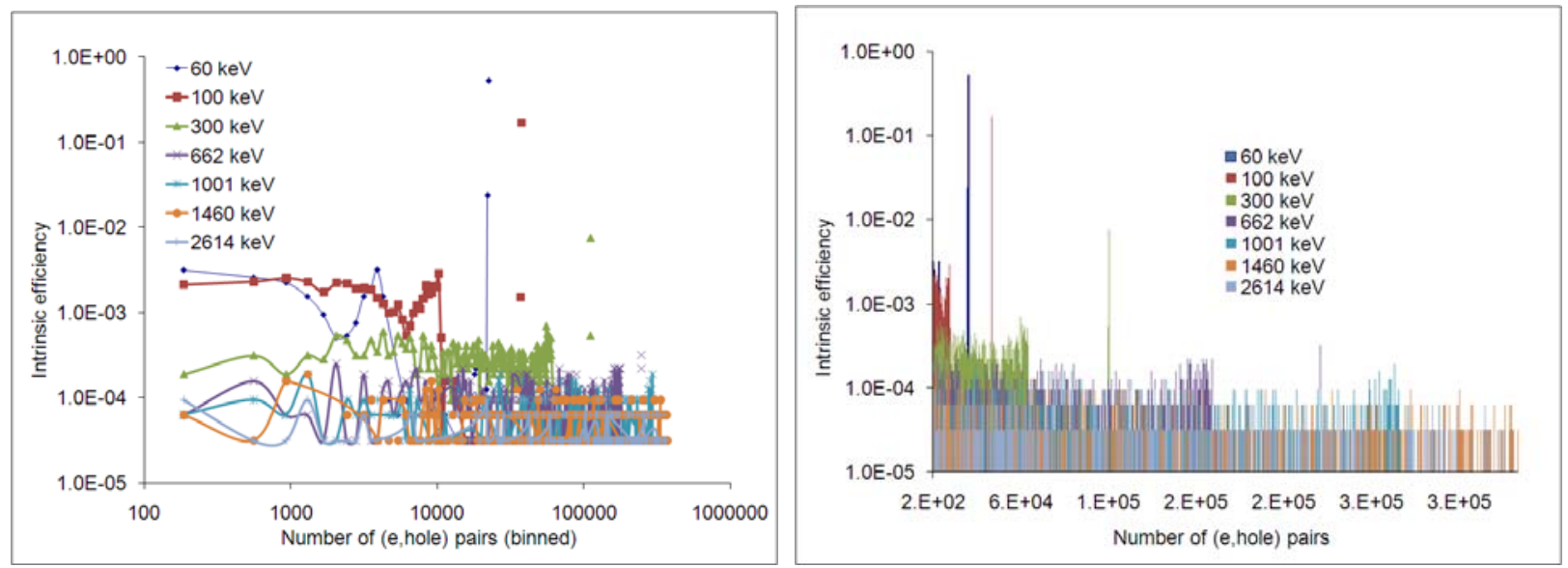

Figure 2: Simulated distributions of pulse amplitudes (i.e. radiation-induced carrier populations) for YIG as determined by Geant4, assuming number of e-h pairs is determined only by the band gap

Depending on the lifetime of the carriers in the material and the carrier generation rate due to the radiation source (and other sources such as thermal), the material could end up in one of three operational limits analogous to other radiation detectors.

A. Low Rate Limit: "Pulse counting mode"

The radiation source is weak and/or the carrier lifetime in the material is very short. In this case the signal in the material has completely or at least substantially diminished by the time the next event reaches the active material. In this way, a set of statistics can be built up of \# of electrons created versus integration time bin. Practically, this would be accomplished by integrating over time steps in the receive radar, looking at frequency and/or amplitude change caused by changes in magnetization or conductivity, for example. Relative lifetimes of $1 \mathrm{~ns}$ could be probed using $1 \mathrm{GHz}$ radiation, $0.1 \mathrm{~ns}$ 
for $10 \mathrm{GHz}$ radiation, etc. It is anticipated that the lifetimes of interest here will be typical of semiconductor trapping times, that is $1 \mu \mathrm{s}(1 \mathrm{MHz})$.

B. High Rate Limit: "Current mode"

Here the radiation source is strong and has perhaps "saturated" the active material. In this case it might be more useful to have a material which did not totally saturate but continued changing its radar/microwave -accessible properties with increasing dose. Here the electrons do not relax fast enough before more are created, and the signal is more or less a cumulative average of the ionization events. The signal is thus proportional to the power deposited in the material, requiring a priori knowledge of either activity or isotope for proper identification. This requirement need is not overtly undesirable, since remote monitoring of spent fuel, for instance, would presumably have some knowledge of what was supposed to be in the canister. The advantage still is that the secondary effect being monitored (radar reflectivity versus frequency or re-radiated power through a harmonic radar) does not require persistence of gamma rays over long distances.

C. Permanent changes: "Integrated dose mode"

It may be that some electromagnetic changes are a) permanent and b) very dose dependent. These types of changes could be used as monitors for exposure. For example, $\mathrm{Bi}_{4} \mathrm{Ge}_{3} \mathrm{O}_{12}$ (BGO) films have shown a linear increase in capacitance after the threshold of $\sim 1.8 \mathrm{mGy}$ was reached [12]. Likewise, many other examples in both ferromagnetic [13] and ferroelectric [14] materials could be given where radiation-induced changes were quasi-permanent until annealed at high temperature.

As previously stated, the output of the Geant 4 model was a material response, called here the "intrinsic efficiency" $\left(F_{\mathrm{i}}\right)$, as a function of energy deposited $\left(E_{\text {dep }}\right.$, by bin) which was converted into number of electron hole pairs $\left(N_{\mathrm{i}}\right)$ through the energy band gap $\left(E_{\mathrm{G}}\right), N_{\mathrm{i}}=E_{\mathrm{dep}} / E_{\mathrm{G}}$. The sum of the intrinsic efficiency over all the bins modeled is always $<1$ since some of the gamma energy passes through the sample, especially at higher energies. The total of all the deposited energy, i.e. the probability of interaction of the gamma ray with the material, was termed the "total intrinsic efficiency" $\left(F_{\text {tot }}\right)$. Furthermore, an additional quantity was computed, corresponding to the average number of electron-hole pairs created per interacting photon, denoted the "expectation value" $\left(N_{\text {avg }}\right)$. This quantity is determined by summing all the pairs of created carriers $\left(N_{\mathrm{i}}\right)$ times binned intrinsic efficiencies $\left(F_{\mathrm{i}}\right)$ and dividing this by the total intrinsic efficiency. This value is then the average number of electrons created for each gamma which deposits at least some of its energy in the material. This pair of quantities, then, gives an indication of the interaction for this specific distance of radiation source and sample size $\left(F_{\text {tot }}\right)$, and in the event of that interaction, the number of electrons created $\left(N_{\text {avg }}\right)$. Representative values for two materials (YIG and CMT) are shown in Table 3 and plotted in Figure 3 as a function of photon energy. The inset shows a blow up of the higher energy region which would be expected to be unaffected by shielding.

$$
N_{\text {avg }}=\frac{\sum_{i} F_{i} N_{i}}{\sum_{i} F_{i}}=\frac{\sum_{i} F_{i} N_{i}}{F_{\text {tot }}}
$$

Table III: Total intrinsic efficiency $\left(\mathrm{F}_{\text {tot }}\right)$ and average number of electrons created $\left(\mathrm{N}_{\text {avg }}\right)$ as a function of incident gamma photon for YIG and CMT; $N_{\text {avg }}$ calculated using the band gap $\left(E_{g}\right)$

\begin{tabular}{llllllll} 
E photon $(\mathrm{keV}):$ & $\mathbf{6 0}$ & $\mathbf{1 0 0}$ & $\mathbf{3 0 0}$ & $\mathbf{6 6 2}$ & $\mathbf{1 0 0 1}$ & $\mathbf{1 4 6 0}$ & $\mathbf{2 6 1 4}$ \\
\cline { 2 - 2 } $\mathrm{F}_{\text {tot }}$ & $56.9 \%$ & $21.7 \%$ & $5.5 \%$ & $3.6 \%$ & $2.9 \%$ & $2.1 \%$ & $1.6 \%$ \\
YIG: $\mathrm{N}_{\text {avg }}$ & $2.2 \mathrm{E}+04$ & $3.0 \mathrm{E}+04$ & $4.2 \mathrm{E}+04$ & $8.9 \mathrm{E}+04$ & $1.4 \mathrm{E}+05$ & $1.5 \mathrm{E}+05$ & $1.4 \mathrm{E}+05$ \\
CMT: $\mathrm{F}_{\text {tot }}$ & $97.6 \%$ & $60.0 \%$ & $8.8 \%$ & $4.6 \%$ & $3.5 \%$ & $2.5 \%$ & $1.4 \%$ \\
CMT: $\mathrm{N}_{\text {avg }}$ & $2.8 \mathrm{E}+04$ & $4.6 \mathrm{E}+04$ & $8.4 \mathrm{E}+04$ & $1.3 \mathrm{E}+05$ & $1.8 \mathrm{E}+05$ & $1.9 \mathrm{E}+05$ & $1.8 \mathrm{E}+05$
\end{tabular}




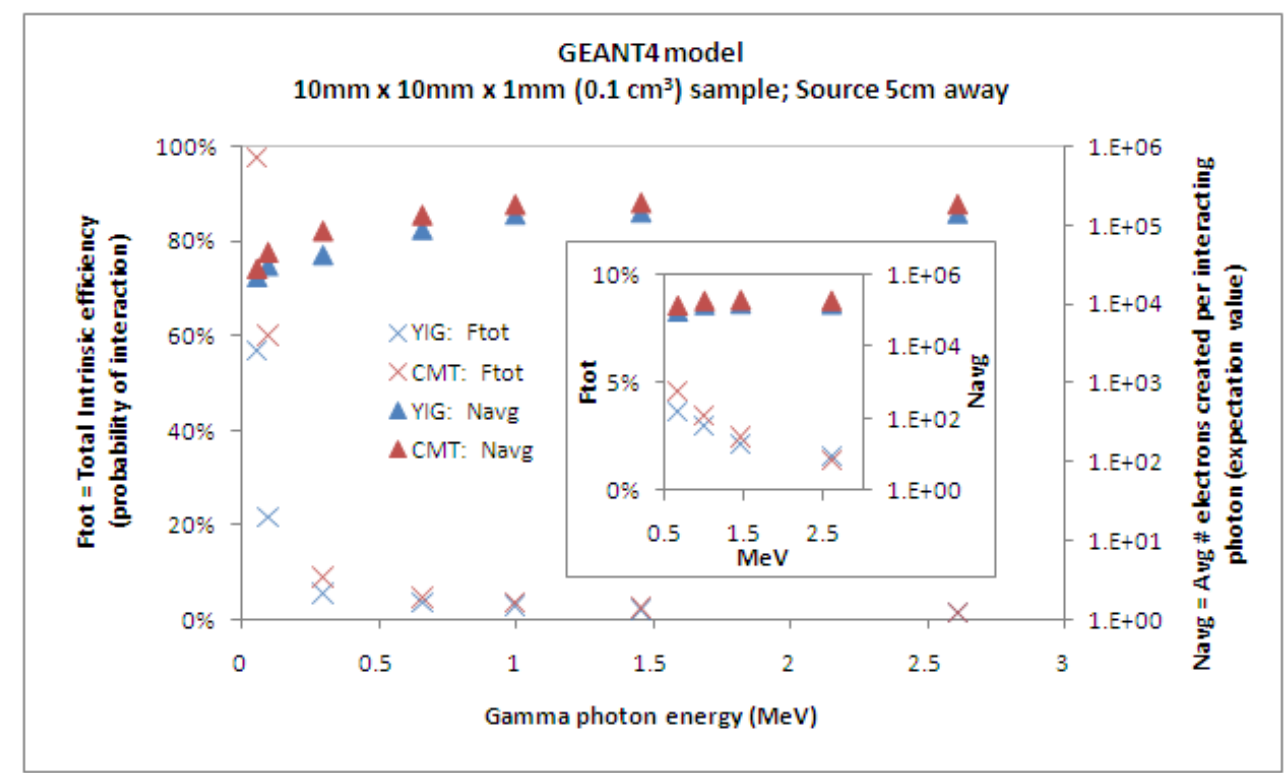

Figure 3: Total intrinsic efficiency $\left(F_{\text {tot }}\right)$ and average number of electrons created $\left(N_{\text {avg }}\right)$ for $Y$ IG and CMT as a function of gamma photon energy; $\mathrm{N}_{\text {avg }}$ calculated using the band gap

Rather than using simply the band gap to determine the number of carriers created, by Devanethan et al [15] have described the average number of carriers in terms of the electron-hole pair production energy $(W)$

$$
\hat{N}=\frac{E_{\gamma}}{W}=\frac{E_{\gamma}(e V)}{2 E_{G}(e V)+1.43(e V)}
$$

The expression for $W$ was determined by fitting experimental data for a number of materials. The difference between $\hat{N}$ and $N_{\text {avg }}$ in our case is just the ratio between $E_{\mathrm{G}}$ and $W$, that is

$$
\hat{N}=N_{\text {avg }} \frac{E_{G}}{W}
$$

It is interesting to note that of all the surveyed materials, the value of $F_{\text {tot }}$ and $N_{\text {avg }}$ (or $\hat{N}$ ) did not vary much (see Table IV for $662 \mathrm{keV}$ data). The intrinsic efficiency can be seen as reflecting the density and average atomic number components of the interaction, while the average number of carriers is primarily affected by the band gap. Since the approximate value of $\hat{N}$ is $10^{4} \mathrm{e}^{-/ p h o t o n}$, and the Geant 4 model assumes $0.1 \mathrm{~cm}^{3}$ of material, we can use as a reasonable estimate of carrier production assuming interaction as $10^{5} \mathrm{e}^{-} /$photon $/ \mathrm{cm}^{3}$.

Table IV: Total intrinsic efficiency $\left(F_{\text {tot }}\right)$, average number of electrons created assuming the energy gap $\left(N_{\text {avg }}\right)$, average number of electrons assuming the pair production energy $(\hat{N})$ for all modeled materials at 662 keV; also shows in the energy gap $\left(E_{G}\right)$, the electron-hole pair production energy $(W)$, and their ratio $\left(E_{G} / W\right)$

\begin{tabular}{lcccccc} 
E photon $(662 \mathrm{keV}):$ & $F_{\text {tot }}$ & $N_{\text {avg }}$ & $E_{\mathrm{G}}(\mathrm{eV})$ & $W(\mathrm{eV})$ & $E_{\mathrm{G}} / W$ & $N^{\wedge}$ \\
\cline { 2 - 7 } YIG & $3.6 \%$ & $8.9 \mathrm{E}+04$ & 2.7 & 6.83 & 0.40 & $3.5 \mathrm{E}+04$ \\
GIG & $4.9 \%$ & $1.2 \mathrm{E}+05$ & 2.5 & 6.43 & 0.39 & $4.5 \mathrm{E}+04$ \\
CMT & $4.6 \%$ & $1.3 \mathrm{E}+05$ & 2.1 & 5.63 & 0.37 & $5.0 \mathrm{E}+04$ \\
LN & $3.3 \%$ & $6.3 \mathrm{E}+04$ & 4 & 9.43 & 0.42 & $2.7 \mathrm{E}+04$ \\
LT & $6.6 \%$ & $9.3 \mathrm{E}+04$ & 3.9 & 9.23 & 0.42 & $3.9 \mathrm{E}+04$ \\
NF & $3.8 \%$ & $1.1 \mathrm{E}+05$ & 2.2 & 5.83 & 0.38 & $4.2 \mathrm{E}+04$
\end{tabular}


The next step in this process would be to take the expected activities of reasonable target materials and multiply their gamma output (usually specified in $\gamma$-photons/sec in $4 \pi$ steradians) and multiply it by the geometric factor to get the number of photons incident on the detector material. This geometric factor $(G F)$ is the exposed area $(A)$ divided by the square of the distance $(d)$ from the detector to the source divided by $4 \pi$ steradians, which for the case studied here is

$$
G F=\frac{A}{4 \pi d^{2}}=\frac{(1 \mathrm{~cm} \times 1 \mathrm{~cm})}{4 \pi(5 \mathrm{~cm})^{2}}=0.00318 \approx 3.2 \times 10^{-3}
$$

Therefore the gamma output for each specific source of interest should be reduced by about three orders of magnitude and this is the activity that should be considered when evaluating the number of electrons created in the detector material. For example, a source of $\sim 10^{8} \gamma$-photons/sec in $4 \pi$ steradians would be seen as $\sim 10^{5} \gamma$-photons/sec (disintegrations per second) by this hypothetical detector, equivalent to an activity of about $10 \mu \mathrm{Ci}$ which is a reasonable laboratory source that could be used for testing. In our assumption of $\sim 10^{5} \mathrm{e}^{-} /$photon $/ \mathrm{cm}^{3}$ created this gives $\sim 10^{10} \mathrm{e}^{-} / \mathrm{cm}^{3} /$ second to cause any transient material changes. Note that this does not yet consider the lifetime of the carriers in the material, which in a typical semiconductor like cadmium zinc telluride $\left(\mathrm{Cd}_{0.9} \mathrm{Z}_{0.1} \mathrm{Te}\right.$ of resistivity $\left.10^{10} \mathrm{ohm}-\mathrm{cm}\right)$ might be $10^{-6}$ seconds [16].

\section{4) Readout mechanisms and system design}

In this section some of the system design considerations relating to the remote readout of detector signal are summarized, including the frequency of operation, intensity and frequency shift modes of operation, and resonator design.

\subsection{SELECTION OF FREQUENCY}

Though the final frequencies for anticipated devices have not been selected, some general guidelines have been established. Radio waves, microwaves, and millimeter waves have very low attenuation in the atmosphere compared to infrared and visible light. The atmosphere is very transparent at wavelengths longer than about $5000 \mu \mathrm{m}$ (at frequencies below $60 \mathrm{GHz}$ ) except for a strong water absorption band about $14000 \mu \mathrm{m}(21-22 \mathrm{GHz})$. Wavelengths shorter than about $32000 \mu \mathrm{m}$ (frequencies above $94 \mathrm{GHz}$ ) have increasingly strong absorption, due to closely spaced water bands, until about $15 \mu \mathrm{m}$ (infrared) where the atmosphere again becomes transmissive. Infrared and visible wavelengths are strongly attenuated by obscurations such as dust, rain, fog, and smoke, whereas radio waves, microwaves, and millimeter-waves are attenuated much less [17].

Atmospheric transmission for 30-300 GHz was calculated using the HITRAN 2008 database for various pathlengths and water contents. It has a minimum frequency input of $1 \mathrm{~cm}^{-1}(10000 \mu \mathrm{m}$ wavelength or 30 $\mathrm{GHz}$ ). The main feature is a strong band centered about $181 \mathrm{GHz}$, with weaker bands appearing at long standoffs and/or increased water vapor. For frequencies shorter than $30 \mathrm{GHz}$, another transmission model can be examined based on Pardo [18]. This model measures water in terms of "precipitable water vapor" (PWV) in millimeters. It is defined as the depth of liquid water that would be formed from condensing all the water in a column of air to liquid water in an area the same as the column of air. So, a $1 \mathrm{~km}$ air column with $1 \%$ volume fraction of air at $300^{\circ} \mathrm{K}$ would give liquid water of a depth of $7.3 \mathrm{~mm}$. PWV values such as 10-30 $\mathrm{mm}$ may be appropriate for near sea level in equatorial or summer temperate conditions. It can be seen that the broad band at $180 \mathrm{GHz}$ remains, but a sharp band at $120 \mathrm{GHz}$ and one at $22 \mathrm{GHz}$ as well as a broad band centered about $60 \mathrm{GHz}$ are evident. The bands at $120 \mathrm{GHz}$ and 60 $\mathrm{GHz}$ are not due to water but due to molecular oxygen, $\mathrm{O}_{2}$. The bottom line of this analysis is that, with the exception of frequencies close to $20 \mathrm{GHz}$ or $60 \mathrm{GHz}$, anything less than $100 \mathrm{GHz}$ will have very low atmospheric attenuation for free space propagation of electromagnetic waves such as would be used for radar. This allows for a considerable amount of design flexibility, since gyromagnetic resonances in ferrite materials, for instance, vary from about $4 \mathrm{GHz}$ to $60 \mathrm{GHz}$ and respond to material tailoring. 


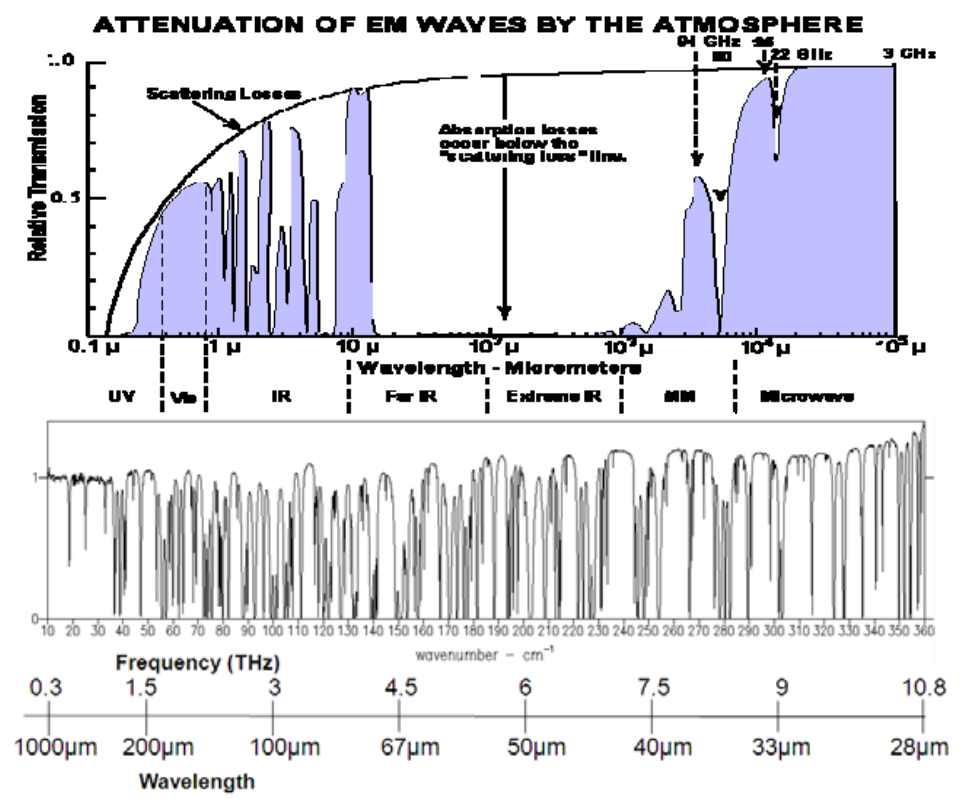

Figure 4: (TOP) Attenuation of electromagnetic radiation by the atmosphere (Wikipedia, "atmosphere absorption"); the blue shaded area indicated transmission; $M M$ indicates millimeter wave region; (BOTTOM) Absorption of a high humidity cell of $10 \mathrm{~cm}$ path length. As can be seen, at frequencies above $300 \mathrm{GHz}$, water accounts for most of the absorption up to $10 \mathrm{THz}$ (also known as the far infrared); after http://sbfel3.ucsb.edu/optical_transport/atm_abs.html

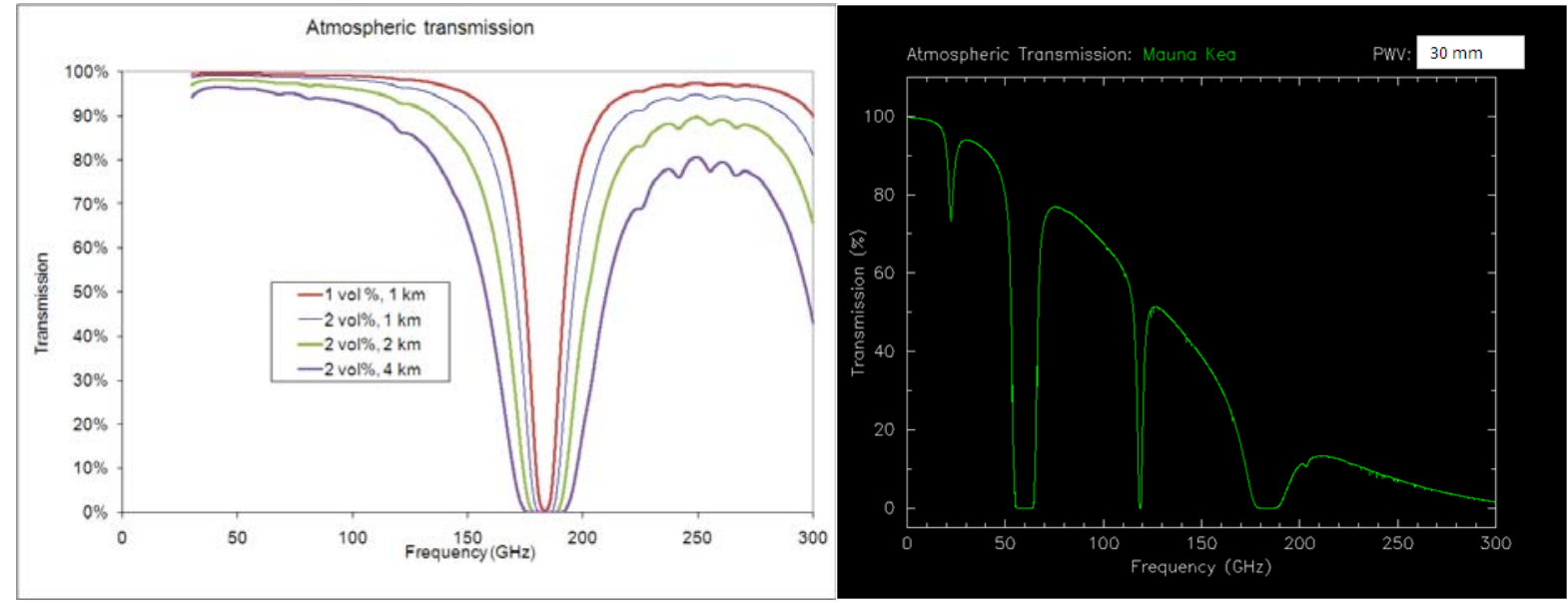

Figure 5: (LEFT) Calculations water absorption in 30-300 GHz using HITRAN 2008 database. The effect of having 1 volume\% of water at $2 \mathrm{~km}$ is identical to having 2 volume\% of water for $1 \mathrm{~km}$. Note: At $300{ }^{\circ} \mathrm{K}$, a $2 \%$ volume fraction of water at $1 \mathrm{~atm}$ corresponds to a relative humidity of $54 \%$; at $20^{\circ} \mathrm{C}$ or $293{ }^{\circ} \mathrm{K}$, at $2 \%$ volume fraction of water at 1 atm corresponds to a relative humidity of 81\%; (RIGHT) Atmospheric transmission from 0 to $300 \mathrm{GHz}$ as computed for the top of Mauna Kea for a precipitable water vapor of $30 \mathrm{~mm}$, showing additional atmospheric absorption bands near $20 \mathrm{GHz}\left(\mathrm{H}_{2} \mathrm{O}\right), 60 \mathrm{GHz}\left(\mathrm{O}_{2}\right)$ and $120 \mathrm{GHz}$ $\left(\mathrm{O}_{2}\right)$. Computed at: http://www.submm.caltech.edu/cso/weather/atplot.shtml

\subsection{INTENSITY SHIFT}

Preliminary PNNL assessments show that power reflectivity changes of $0.05 \mathrm{~dB}$ are detectable by microwave/millimeter-wave transceivers. This amount of power change corresponds to a $1 \%$ change in 
reflectivity.

$$
R=\left(\frac{\eta^{*}-1}{\eta^{*}+1}\right)^{2}=\left(\frac{\sqrt{\mu^{*} / \varepsilon^{*}}-1}{\sqrt{\mu^{*} / \varepsilon^{*}}+1}\right)^{2}=\left(\frac{\sqrt{\mu^{*}}-\sqrt{\varepsilon^{*}}}{\sqrt{\mu^{*}}+\sqrt{\varepsilon^{*}}}\right)^{2}=\left(\frac{\sqrt{\mu^{\prime}+i \mu "}-\sqrt{\varepsilon^{\prime}+i \varepsilon "}}{\sqrt{\mu^{\prime}+i \mu^{\prime \prime}}+\sqrt{\varepsilon^{\prime}+i \varepsilon "}}\right)^{2} \approx\left(\frac{\sqrt{\mu^{\prime}}-\sqrt{\varepsilon^{\prime}}}{\sqrt{\mu^{\prime}}+\sqrt{\varepsilon^{\prime}}}\right)^{2}
$$

Power reflectivity ( $R$, above) of a surface in contact with air is a function of wave impedance $(\eta)$. Materials with significant conductivity have a slightly different form for the impedance than shown here in the simplified case ignoring losses (imaginary components). Changes in the real part of the permittivity $\left(\varepsilon^{\prime}\right)$ and the real part of the permeability $\left(\mu^{\prime}\right)$ affect reflectivity and are altered by the freecarrier population. For the case of a material where ionizing radiation only causes a change in its electric permittivity $(\varepsilon)$, the need to create a $1 \%$ change in reflectivity requires a $6 \%$ change in the dielectric constant (e.g., $\varepsilon^{\prime}=9.4 \rightarrow 10$, or $\Delta \varepsilon^{\prime} / \varepsilon^{\prime} \approx 6 \%$ ). As stated above, there are materials that display a higher sensitivity to ionizing radiation due to changes in their permeability. For example, looking at permeability changes only, one can consider YIG. Taking the magnitude of the relative real permeability of demagnetized YIG at $1 \mathrm{GHz}$ as 0.98 [19], its real dielectric constant as 12.5 [20], and neglecting dielectric and magnetic losses, a change of only $6 \%$ in permeability (decrease to 0.92 ) is required to see the $1 \%$ reflectivity change required. This change is much more reasonably achievable than a large change in the real permittivity (9.4 to 10) previously mentioned.

\subsection{FREQUENCY SHIFT}

It is necessary to know what frequency shift could be seen by an interrogating microwave/millimeter wave system. Center frequency drift or change could be measured to better than $<10 \mathrm{kHz}$ precision with a simple swept spectrum analysis using standard HP spectrum analyzers. The stability of these measurements is set by the quality of the set-up and freedom of movement or, sometimes a change of temperature of the system. It is possible to achieve higher resolution with care and better signal analysis techniques. In any event, these are much smaller numbers than the typical linewidth as shown above $(0.03$ to $0.85 \mathrm{GHz}$ ).

For resonant cavities such as dielectric loaded split-ring resonator (SRRs), one is actually seeing a structure resonance with the fundamental width set by the loss tangent (which could also include magnetic losses) of the loaded material. The DC properties of the loaded dielectric (or magnetodielectric) determine the center frequency of the structure. In the case of well made, pure samples, the loss tangents are quoted as being $<0.001$ typically, and one can see resonance dips in the SWR (standing wave resonance) reflected $\mathrm{RF}$ power with widths $\sim 200 \mathrm{KHz}$ to $2 \mathrm{MHz}$ at SRR resonance center frequencies $\mathrm{f}_{0} \sim 0.3$ to $3 \mathrm{GHz}$. Typically, the resonant $\mathrm{Q}$ factor defined roughly as $\mathrm{f}_{0} / \Delta \mathrm{f}(-6 \mathrm{~dB})$ of $>1,000$ to 3,000. Theoretically the Q-factor could be as high as 10,000 , but we saw no evidence we could reach this value with the tolerances of construction and fit that we achieved in the 1990s in dielectric loaded SRRs. This SWR resonance width was set by many factors, but chief among them was the quality of the SRR rings' fit to the loaded dielectrics and the loss tangents of real samples. Typically manufacturers state excellent loss tangents, but in practice, even for single crystals with excellent tolerance of construction and polished finish, we saw that the loss tangents (now the limiting factor - not crystal fit) were seldom as low as advertised. We think we can make the SRR cavities quite well so that theoretical limits of 10,000 could be achieved, but we would be surprised to see such high Q-factors with multi-domain crystals. That being said, Q factors of 1,000 are probably adequate for seeing the shifts predicted here.

\subsection{RESONANT CAVITIES AND RESONANT CIRCUITS}

In this section, it is shown that for one kind of resonant cavity, the split ring resonator (SRR), the structural resonance changes substantially with a small permeability change. 
For one particular kind of resonator, the split-ring resonator described in [21] has a resonant frequency $f_{0}$ in terms of inductance $\mathrm{L}$ and capacitance $\mathrm{C}$ of:

$$
\begin{aligned}
& f_{0}=\frac{1}{2 \pi} \frac{1}{\sqrt{L C}}=\frac{1}{2 \pi}\left(\frac{c}{r}\right) \sqrt{\frac{d}{\pi w K}} \\
& K=\left(\frac{\varepsilon}{\varepsilon_{0}}\right)\left(\frac{\mu}{\mu_{0}}\right)=\varepsilon_{r} \mu_{r}
\end{aligned}
$$

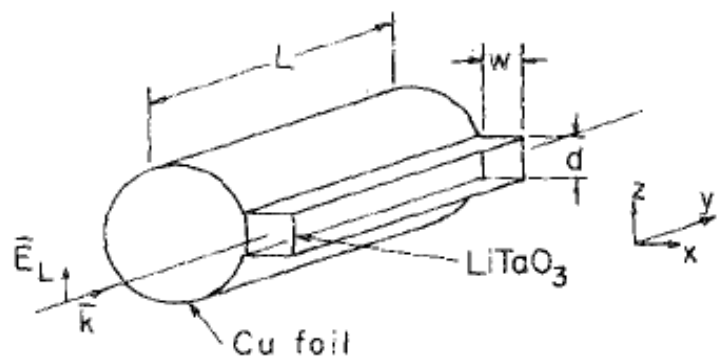

Figure 6: Schematic of inner dimensions of a split ring resonator, after [21]

Where $w$ is the width and $d$ the depth of the slot, $r$ is the radius of the bore, and $c$ is the speed of light. Note that the slot of the SRR can be filled with any dielectric material (including air) with a relative permittivity $\varepsilon_{\mathrm{r}}$, and the bore of the SRR can be filled with a magnetic material with relative permeability $\mu_{\mathrm{r}}$. Thus the resonant frequency of the SRR depends on the size of the slot, the permittivity of the material in the slot, and the permeability of the material in the bore.

Assume $w / d=1$ and $r=0.1895$ ", slot is loaded with Teflon $\left(\varepsilon_{\mathrm{r}}(1-10 \mathrm{GHz})=2.2\right)$, and bore is loaded with YIG $\left(\mu_{\mathrm{r}}(1 \mathrm{GHz})=0.2\right.$, demagnetized, below resonance, $\mu_{\mathrm{r}}(10 \mathrm{GHz})=1$, demagnetized, above resonance [19]). Calculating the resonant frequency of the SRR at these two limits gives the cavity frequency resonance change at 8.44 to $3.77 \mathrm{GHz}$. This is a large change in resonant frequency due to the rapidly changing permeability of YIG in this frequency range due to its material gyromagnetic resonance frequency (GMRF). It is anticipated that an SRR of this type will be highly sensitive to the location of the material GMRF.

A similar analysis was performed using a permittivity change, based on work done for neutron irradiated lead zirconium titanate (PZT) films [14]. Here there is no resonance assumed, just a change in the magnitude of the real value of the relative permittivity. Values from the cited paper were fitted and extrapolated from $1 \mathrm{MHz}$ to $10 \mathrm{GHz}$. Computed change in the SRR resonant frequency was about 100 $\mathrm{MHz}$ which is very easy to resolve. Note that this is an integrated permanent change in the material permittivity due to the radiation.

The split ring resonator concept is good for laboratory demonstrations, but may not be reasonable for a deployable solution. In that case a resonant circuit may be more appropriate. As has been shown by a recent invention, tank circuits (or RLC resonant circuits) can be employed as radiation detectors [22]. Theoretically, a device could operate as a "single event" detector using a semiconductor element whose resistivity decreased with irradiation, or as a "total dose" detector where over time the semiconductor accumulates enough defects that its resistivity increases. Depending on whether the RLC circuit is a series or parallel circuit, the damped resonant frequency will increase or decrease with resistance increase.

Tank circuits can also be useful for determining magnetic susceptibility (permeability) of materials. Inserting a magnetic sample in an LC resonator causes a shift in the resonant frequency which is proportional to the magnetic susceptibility of the sample [23]. More precisely, the change in inductance 
is proportional to the change in impedance, which is itself proportional to the square root of the product of the magnetic permeability and the resistivity [24].

Some of the following is based on Pozar [25]. The resonant frequency $\omega_{0}$ is dependent on both the inductance $\mathrm{L}$ and capacitance $\mathrm{C}$. The damping factor $\zeta$ is the ratio of the attenuation to the resonant frequency. The Quality factor ( $\mathrm{Q}$ factor) describes the sharpness of the resonance, and is the ratio of the resonant frequency to its bandwidth, measured at the half power frequencies $\omega_{1}$ and $\omega_{2}$.

Capacitive effects could be induced by radiation by changes in the dielectric permittivity and/or charge transfer conduction. Changes in the inductance would be caused by magnetic permeability changes in the material. Both capacitance and inductance depend on geometry of the component and on the electromagnetic properties (permittivity, permeability), and are directly proportional to these electromagnetic properties, hence their alternative terms - dielectric permittivity = capacivity, magnetic permeability $=$ inductivity. The effect of radiation on an RLC tank circuit is one way to provide sensitivity to a harmonic radar transponder described below.

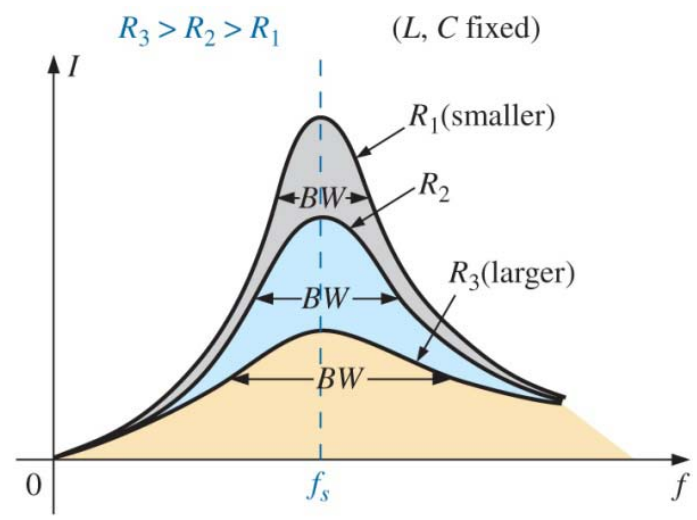

(a)

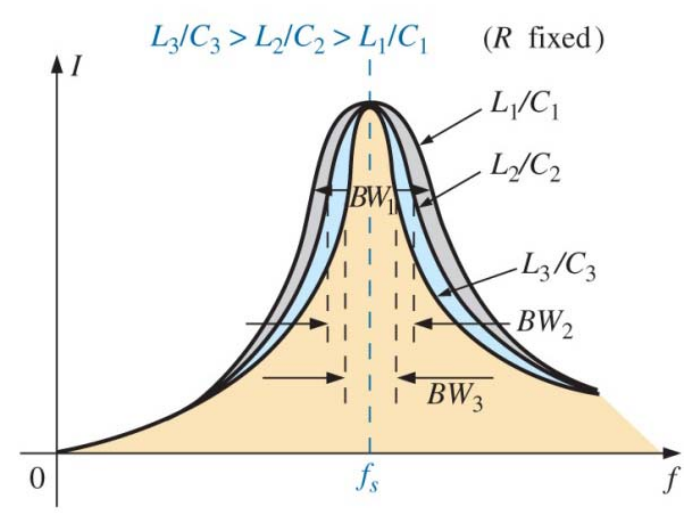

(b)

Figure 7: Effect of $\mathbf{R}$ and $\mathrm{L} / \mathrm{C}$ on the series resonance. Larger $\mathrm{R}$ leads to broader and weaker transmission windows. Smaller $\mathrm{L} / \mathrm{C}$ leads to larger bandwidths and less selectivity.

\subsection{HARMONIC RADAR}

To improve standoff sensing using passive radiation sensors, harmonic radars can be used to eliminate backscatter problems from other objects in its field of view. To overcome backscatter from other objects that may surround the transponder, harmonic radar can be employed in such a way that the fundamental signal is transmitted and the second harmonic of the signal is reradiated by the harmonic tag. Figure 8 shows the system architecture of the Harmonic Radar configuration. There is a non-linear junction device in the transponder that produces the second harmonic and coupled to the radiation-sensitive circuit. Objects that surround the tag will reflect only the fundamental signal which would tend to mask the signals from the transponder if only the fundamental frequency was reradiated. However with the second harmonic reradiated, the harmonic receiver will readily detect the signal from the transponder and reject the fundamental signals.

The following analysis is based on Riley and Smith [26], where a harmonic radar system for tracking honeybees was described. In their application, they transmitted at $9.4 \mathrm{GHz}(\lambda=32 \mathrm{~mm})$ and received the harmonic at $18.8 \mathrm{GHz}(\lambda=16 \mathrm{~mm})$. It can be shown that the maximum range (in meters) for a harmonic radar system is: 


$$
R_{\mathrm{m}}=\left\{\left(E_{\mathrm{dh}} \times A_{2} \times E_{\mathrm{a} 2} \times G_{\mathrm{f}} \times P_{\mathrm{t}} \times G_{\mathrm{dh}} \times A_{\mathrm{df}}\right) /\left(L_{\mathrm{gh}} \times L_{\mathrm{gf}} \times(4 \pi)^{2} \times P_{\mathrm{m}}\right)\right\}^{0.25} \mathrm{~m}
$$

Where $\mathrm{Pm}$ is the minimum received power required for detection (in Watts), given by

$$
P_{\mathrm{m}}=k \times T \times B \times 10^{6} \times N_{\mathrm{f}} \times V_{\mathrm{f}} \mathrm{W}
$$

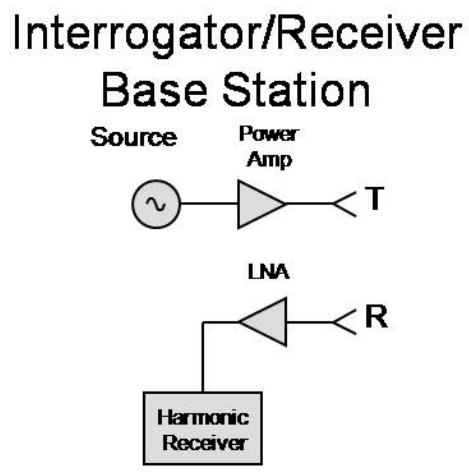

\section{Harmonic Tag}

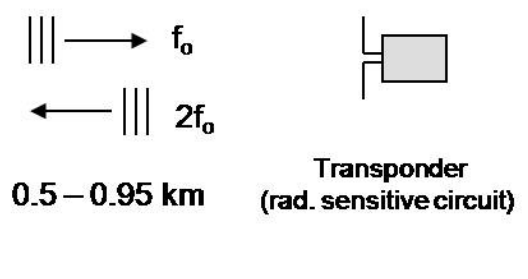

Figure 8: System architecture of the harmonic radar configuration; LNA is a low noise amplifier

Where $\mathrm{k}$ is Boltzman's constant, $\mathrm{T}$ is the temperature in Kelvin, and the other parameters are described below. The range realized in their harmonic radar was about 1 kilometer, which could be substantially improved for a different antenna design as would be appropriate for our application. The rationale for going through this detail was to investigate the feasibility and potential for improvement of the individual factors in the harmonic radar system and to identify the parameters which impact the system performance. It is proposed that such as system could be made with a radiation-sensitive component (experiencing a change in capacitance, resistance, or inductance) in the circuit of the on-board transponder (i.e. unpowered "tag" which responds to the interrogating signal). The frequency shift in the expected receive signal would be an indication of radiation, and would be detected by using a swept frequency receiver.

**Note that designing a more appropriate antenna would greatly increase the range.

\begin{tabular}{|l|l|l|}
\hline & Description of parameter & Value in Riley and Smith [26] for bee transponder \\
\hline $\mathrm{R}_{\mathrm{m}}$ & Maximum range & 0.5 to 0.95 kilometers \\
\hline $\mathrm{E}_{\mathrm{dh}}$ & Power conversion to $2^{\text {nd }}$ harmonic & $0.8 \%$ \\
\hline $\mathrm{A}_{2}$ & Aperture of receive antenna (harmonic) & $0.46 \mathrm{~m}^{2}$ \\
\hline $\mathrm{E}_{\mathrm{a} 2}$ & Efficiency of receive antenna (harmonic) & 0.65 \\
\hline $\mathrm{G}_{\mathrm{f}}$ & Gain of transmit antenna & $14,454(41.6 \mathrm{~dB})$ \\
\hline $\mathrm{P}_{\mathrm{t}}$ & Transmitter output power & $25 \mathrm{~kW}$ \\
\hline $\mathrm{G}_{\mathrm{dh}}$ & Gain of receive antenna (harmonic) & $2.31(3.64 \mathrm{~dB}) ;$ broadside-on gain of full wave dipole \\
\hline $\mathrm{A}_{\mathrm{df}}$ & Effective aperture of half-wave dipole antenna & $\begin{array}{l}1.33 \mathrm{x} 10^{-4} \mathrm{~m}^{2} ; \text { calculated from the broadside-on gain of } \\
\text { half wave dipole and the wavelength** }\end{array}$ \\
\hline $\mathrm{L}_{\mathrm{gh}}$ & $\begin{array}{l}\text { Loss in the waveguide from the receive antenna } \\
\text { to the receiver }\end{array}$ & $1.58(2 \mathrm{~dB})$ \\
\hline $\mathrm{L}_{\mathrm{gf}}$ & $\begin{array}{l}\text { Loss in the waveguide from the transmitter to } \\
\text { the transmit antenna }\end{array}$ & $1.17(0.7 \mathrm{~dB})$ \\
\hline $\mathrm{B}$ & Receiver bandwidth & $20 \mathrm{MHz}$ \\
\hline $\mathrm{N}_{\mathrm{f}}$ & Noise figure of receiver amplifier & $2.4(3.8 \mathrm{~dB})$ \\
\hline $\mathrm{V}_{\mathrm{f}}$ & Visibility factor (false alarm rate) & $10(10 \mathrm{~dB})$ \\
\hline
\end{tabular}




\section{5) Mechanism of material interaction}

Only one possible physical mechanism for radiation-induced change is discussed below. Others, such as the effect of neutrons and other radiation on ferroelectric materials (e.g. [7, 8, 27, 28]), is currently being researched. The photomagnetic effect, discussed below, was heavily investigated starting in the mid1970 's for potential application to magneto-optic recording.

\subsection{THE PHOTOMAGNETIC EFFECT}

The photomagnetic effect is generally understood as originating from a charge transfer between multivalent cations (such as $\mathrm{Fe}, \mathrm{Cr}, \mathrm{Mn}$, or $\mathrm{Cu}$ ) which causes a change in magnetocrystalline anisotropy field and a pinning of magnetic domain walls. Considerable research went into this effect in the early days of magnetic recording, particularly at Philips in the Netherlands, but research had slowed considerably by the mid-1970s. There is still some active research going on, however, searching for materials where the effect is observable and persistent at room temperature $[29,30]$.

Nagaev [3] explains the phenomena of the photomagnetic effect (PME) differently, referring to a quasiparticle called a ferron which consists of an electron and a ferromagnetic microregion. Rather than being simply a charge transfer of one electron between $\mathrm{Fe}^{2+}$ and $\mathrm{Fe}^{3+}$, quantum mechanically the extra charge is spread out over multiple sites, thus facilitating the creation of microdomains. There can be multiple physical means for photomagnetism in semiconductors, depending on the type (degenerate, nondegenerate), state of the light (circular polarized, plane polarized, unpolarized). Circularly polarized light affects the exchange while plane polarized light affects the anisotropy. Photomagnetism can come from free photoelectrons (small effect on the order of $0.2 \mathrm{~K}$ shift of Tc and requires about $10^{18} \mathrm{e}^{-} / \mathrm{cm}^{3}$ ), trapped electrons at local centers where traps are filled then free photoelectrons are generated (larger effect), or precipitation of insulator-to-metal phase transitions. In some materials, the metal-insulator transition is related to photomagnetism, where the insulating ferron state (ferromagnetic sphere in an antiferromagnetic matrix) converts to a conductor (anti-ferromagnetic sphere in ferromagnetic matrix) by increasing the temperature or the field. Trapped electrons can cause lattice constant changes which affect the $\mathrm{d}-\mathrm{d}$ or $\mathrm{f}-\mathrm{f}$ electron exchange energy and increase the local ferromagnetism, and magnetization increases with illumination. If the binding energy is small for electrons at traps, they are not really localized but "smeared out" over many sites promoting indirect exchange. This model is in contrast to the fixed position and valency exchange model proposed above.

For a demagnetized ferrite such as a garnet or spinel or hexaferrite, the gyromagnetic resonance frequency (GMRF) occurs in the microwave to millimeter wave region $(\sim 3$ to $60 \mathrm{GHz})$ and is directly proportional to the magnetocrystalline anisotropy field. Near the GMRF the magnetic permeability undergoes a resonance where it rapidly increases when approaching it from low frequencies then oscillates and goes below unity at frequencies above the GMRF (see Figure 9a). It is this anisotropy field which is expected to be changed by radiation, resulting in a change of the permeability by a substantial percentage given its large slope over a narrow frequency range near the resonance. In real materials the loss (imaginary part) is not as sharp as shown in Figure 9a because there are other mechanisms contributing to the loss at lower frequencies which affects the shape of the real part of the permeability and is also dependant on magnetization state (see Figure $9 \mathrm{~b}$ for demagnetized $\mathrm{Ca}-\mathrm{V}$ garnet). Ideally, the different contributions can be separated out into low frequency effects (domain wall relaxations, movement, rotations) and high frequency effects (spin or gyromagnetic resonance) (see Figure 9c). These various effects occur at different frequencies in the microwave/ millimeter-wave depending on the material, so it is desirable to have knowledge of the whole permeability spectrum out to $\sim 100 \mathrm{GHz}$ especially for hexaferrites. 
Predictions of magnetic permeability as a function of frequency have become increasingly sophisticated, now including effects of domain and grain demagnetization factors present in demagnetized polycrystalline ferrites [31]. Robust solutions for the complex permeability of single crystals which are magnetically saturated have been around for a long time [32]. It is anticipated that a model could be created predicting the permeability around the resonance. Models for the permeability above the resonance frequency are well established but those for below the resonance frequency are more complicated and less robust, particularly for demagnetized and partially magnetized materials. Models of this nature are needed to predict the permeability spectrum near the resonance to aid in understanding the physical effect of radiation on magnetic materials.
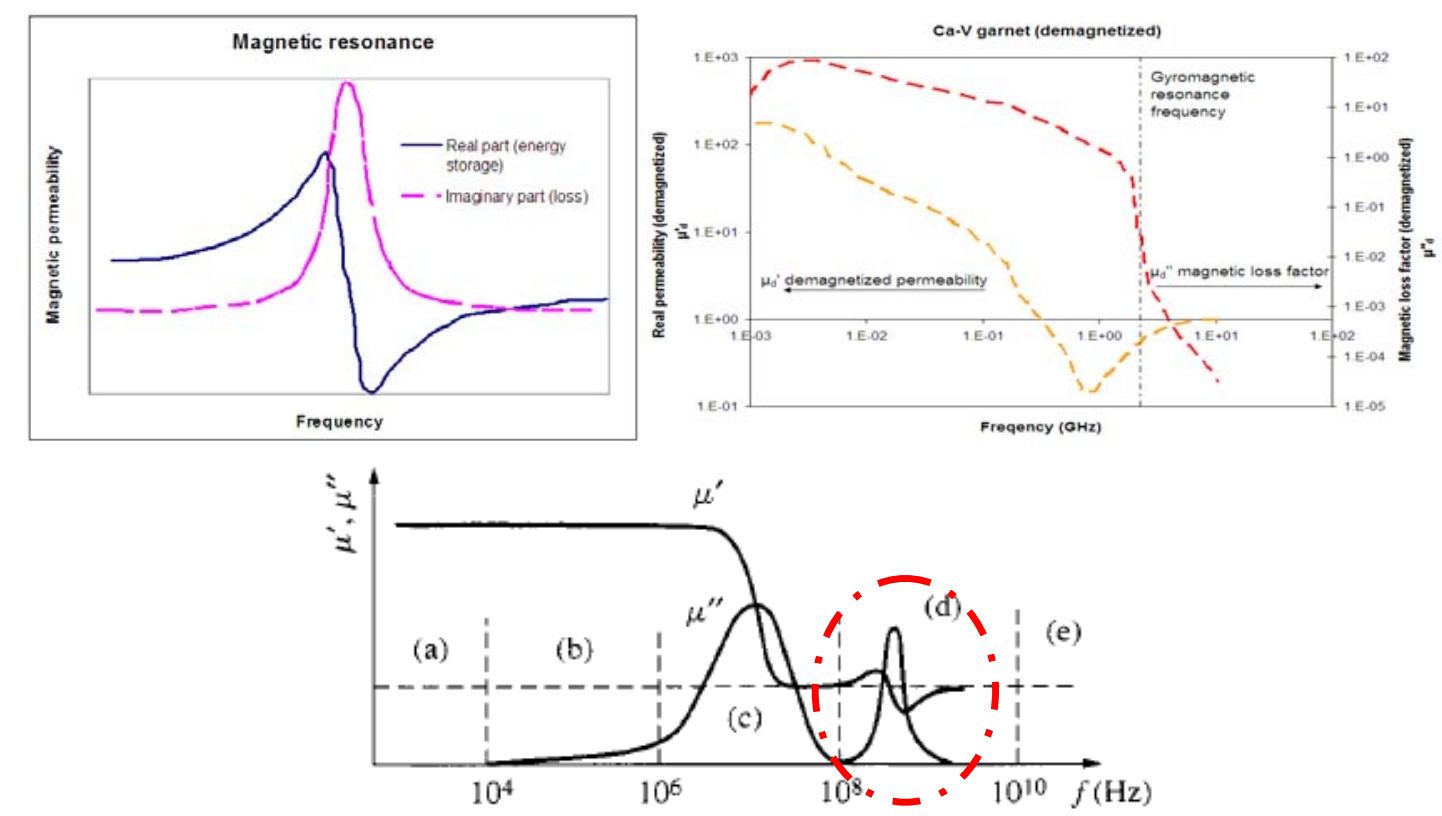

Figure 9: (a,UL) Simple schematic of gyromagnetic resonance. (b,UR) Frequency dependence of the real and imaginary components of the demagnetized permeability of $\mathrm{Ca}-\mathrm{V}$ Garnet with no applied magnetic field. Data from Krupka and Geyer [19]. Lower frequency "low field" losses are associated with having magnetic domains with opposite magnetization directions [31]. (c,below) Idealized permeability spectrum of magnetized ferrite showing low frequency losses due to domain wall motion and high frequency losses due to spin resonance (circled) [33].

\section{6) Interim conclusions, unanswered questions, and future plans}

Based on the studies performed to date, it seems that radiation detection can be achieved by using frequency dependent absorption due to material resonances (e.g. gyromagnetic resonances, ferroelectric grain resonances) and/or structural resonances (in split ring resonators, other resonant cavities). The documentation of the photomagnetic effect in the literature lends support for a change in permeability and resonant frequency with infrared, visible, x-ray, and gamma-ray excitation. There is likely a similar effect for the electric field, and there is some evidence in the literature that piezoelectrics undergo huge reductions in permittivity due to neutron irradiation [14]. It seems feasible also that neutron scattering would produce changes in magnetization and/or polarization through perturbation of the electronic structure and change of the magnetocrystalline anisotropy, for example.

Knowledge of the time constant for these processes has the most uncertainty at this point. In the literature the photomagnetic effect was investigated for its quasi-permanent nature at low temperatures (below about $130 \mathrm{~K}$ for YIG). Relaxation of the radiation-induced changes was "rapid" at room temperatures but 
was not measured. This rapid thermalization of the effect could be beneficial for a pulse-counting type mode. More detailed predictive models of magnetization/ polarization change based on the finding here of about $10^{5} \mathrm{e}^{-/ \text {photon }} / \mathrm{cm}^{3}$ created is warranted. Our conclusion from our preliminary modeling efforts and extensive literature search is that the concept warrants the collection of experimental data to further refine the understanding of the utility of electromagnetic materials for remote detection of radiation.

Demonstrations need to be performed still on real devices. These are planned as part of this feasibility project. Measurements will be taken in the split ring resonators with excitation by arc lamps and/or sealed sources. 


\section{7) References}

1 Tepper, G. and J. Losee, "Detection of single photon ionization events using a contactless microwave technique," Review of Scientific Instruments, 68(1), 55-57 (1997).

2 Metselaar, R. and M.A.H. Huyberts, "Influence of dopants on photomagnetic effects in YIG," Philips Research Reports, 29, 453-475 (1974).

3 Nagaev, E.L., "Photoinduced magnetism and conduction electrons in magnetic semiconductors," Phys. Stat. Sol. (a), 145, 11-64 (1988).

4 Teale, R.W., "Device for detecting and recording electromagnetic radiation including an active magnetic element a parameter of which can be changed by radiation," USPTO 3,681,602, (1972).

5 Kim, S.-S., Y.-C. Yoon, and K.-H. Kim, "Electromagnetic Wave Absorbing Properties of HighPermittivity Ferroelectrics Coated with ITO Thin Films of $377 \mathrm{ohm}, "$ Journal of Electroceramics, 10(95-101) (2003).

6 Xi, Y., H. McKinstry, and L.E. Cross, "The Influence of Piezoelectric Grain Resonance on the Dielectric Spectra of $\mathrm{LiNbO}_{3}$ Ceramics," Journal of the American Ceramic Society, 66(9), 637641 (1983).

$7 \quad$ Sternberg, A.R., et al., "Radiation effects in transparent ferroelectric ceramics," Proc. SPIE 2967, Optical Inorganic Dielectric Materials and Devices, 186-192 (1997).

8 Kulikov, D.V., et al., "Computer simulation of ferroelectric property changes in PLZT ceramics under neutron irradiation," Proc. SPIE 4348, Fourth International Workshop on Nondestructive Testing and Computer Simulations in Science and Engineering, 264-269 (2001).

$9 \quad$ Wolfbeis, O.S., "Sensor Paints," Advanced Materials, 20, 3759-3763 (2008).

10 Agostinelli, S., "Geant4: A Simulation Toolkit," Nuclear Instruments and Methods in Physics Research Section A, 506, 250 (2003).

11 Gao, F., et al., "Electron-hole pairs created by photons and intrinsic properties in detector materials," IEEE Transactions in Nuclear Science, 55, 1079 (2008).

12 Arshak, K., et al., "Properties of BGO thin films under the influence of gamma radiation," Thin Solid Films, 516(7), 1493-1498 (2008).

13 Parkhomenko, V.D., et al., "Peculiarities of Radiation Damage in Ferrites with Spinel Structure," Physica Status Solidi A, 38, 57-66 (1976).

14 Sternberg, A.R., et al., "Irradiation effects in lead zirconate thin films," Proc. SPIE 5122, Advanced Organic and Inorganic Optical Materials, 341-347 (2003).

15 Devanathan, R., et al., "Signal variance in gamma-ray detectors--A review," Nuclear Instruments and Methods in Physics Research Section A: Accelerators, Spectrometers, Detectors and Associated Equipment, 565(2), 637-649 (2006).

16 Tepper, G.C., et al., "Contactless measurements of charge traps and carrier lifetimes in detectorgrade cadmium zinc telluride and mercuric iodide," Proc. SPIE 4141, Hard X-Ray, Gamma-Ray, and Neutron Detector Physics II, 76-88 (2000).

17 Harris, D.C., Materials for Infrared Windows and Domes: Properties and Performance. SPIE Press (1999).

18 Pardo, J.R., J. Cernicharo, and E. Serabyn, "Atmospheric transmission at microwaves (ATM): an improved model for millimeter/submillimeter applications," Antennas and Propagation, IEEE Transactions on, 49(12), 1683-1694 (2001).

19 Krupka, J. and R.G. Geyer, "Complex permeability of demagnetized microwave ferrites near and above gyromagnetic resonance," Magnetics, IEEE Transactions on, 32(3), 1924-1933 (1996).

20 Sirdeshmukh, L., et al., "Dielectric properties and electrical conduction in yttrium iron garnet (YIG)," Bulletin of Materials Science, 21(3), 219-226 (1998).

21 Kelly, J.F. and A. Gallagher, "Efficient electro-optic modulator for optical pumping of Na beams," Review of Scientific Instruments, 58(4), 563-566 (1987). 
22 Burger, R., et al., "Radiation Detector having All-Metal Circuitry Operation of which is based on Electron Spin," USPTO 7,220,968, (2007).

23 Clover, R.B. and W.P. Wolf, "Magnetic Susceptibility Measurements with a Tunnel Diode Oscillator," Review of Scientific Instruments, 41(5), 617-621 (1970).

24 Srikanth, H., J. Wiggins, and H. Rees, "Radio-frequency impedance measurements using a tunnel-diode oscillator technique," Review of Scientific Instruments, 70(7), 3097-3101 (1999).

25 Pozar, D.M., Microwave Engineering. 3rd ed., John Wiley \& Sons (2005).

26 Riley, J.R. and A.D. Smith, "Design considerations for an harmonic radar to investigate the flight of insects at low altitude," Computers and Electronics in Agriculture, 35, 151-169 (2002).

27 Lesnyh, D.A., et al., "Computational study of the influence of oxygen vacancies on the polarization in irradiated and annealed PLZT ceramics," Proc. SPIE 4627, Fifth International Workshop on Nondestructive Testing and Computer Simulations in Science and Engineering, 170-176 (2002).

28 Lesnyh, D.A., et al., "Changes in the temperature dependence of the dielectric constant in irradiated antiferroelectric thin films," Proc. SPIE 5127, Sixth International Workshop on Nondestructive Testing and Computer Simulations in Science and Engineering, 136-139 (2003).

29 Davidenko, I.I., "Photoinduced magnetization reversal in yttrium-iron garnets doped with silicon and cobalt," Journal of Alloys and Compounds, 369(1-2), 166-169 (2004).

30 Bettinger, J.S., R.V. Chopdekar, and Y. Suzuki, "Room temperature photoinduced magnetization of manganese zinc ferrite," Applied Physics Letters, 94(7), 072505-3 (2009).

31 Gelin, P., P. Queffelec, and F. Le Pennec, "Effect of domain and grain shapes on the dynamical behavior of polycrystalline ferrites: Application to the initial permeability," Journal of Applied Physics, 98(5), 053906-6 (2005).

32 Baden Fuller, A.J., Ferrites at microwave frequencies, IEEE Electromagnetic Waves Series, ed. P.J.B. Clarricoats, E.D.R. Shearman, and J.R. Wait. Vol. 23. Peter Peregrinus,London (1987).

33 Chen, L.F., et al., Microwave Electronics: Measurement and Materials Characterization. John Wiley \& Sons (2004). 